\title{
Evidence of Hnf1a Gene in Invertebrates: The Echinodermata
}

\author{
Michel Leclerc \\ 556 rue Isabelle Romée, 45640 SANDILLON (FRANCE) \\ *Corresponding Author: Michel Leclerc, 556 rue Isabelle Romée, 45640 SANDILLON (FRANCE).
}

\begin{abstract}
HNF1A gene we met usually in Human was discovered for the first time in Invertebrates, in Echinodermata. Its parameters, its sequence in 5'-3' was clearly demonstrated in Ophiocomina nigra (Ophuirids) but not in Antedon bifida (Crinoïds) (e-value not significant enough). In human HFNIA gene seems in relation to liver and pancreas.
\end{abstract}

Keywords: Invertebrates; Echinodermata ; Ophuirids ; HNF1A Gene.

\section{INTRODUCTION}

HNF1A(HNF1 Homeobox A gene) is a typical one we met usually in human on chromosome 12.The protein encoded by this gene is a transcription factor required for the expression of several liver-specific genes. It is also ubiquitously expressed in many tissues and cell types Since we discovered Invertebrate Primitive Antibody( Ref.1-2) and invertebrrate lymphocytes in Echinodermata, we decide to look for genes and cells which are implicated in » showing the antigen » and may be in different cell types.

It is the main reason we tried to discover HNF1A gene in Echinodermata. Ophuirid and Crinoïd genomes were studied.

\section{Materials And Methods}

Animals: Ophiocomina nigra (Ophuirid) Antedon bifida(Crinoïd) were obtained at the station «Of Biologie Marine of Roscoff » France.

Obtention of ophuirid and crinoïd mRNA: Digestive coeca were excised from their bodies and mRNA were obtained from Uptizol (Interchim) then quality controls were operated.

Sequencing : Sequencing was made on Illumina Next Seq 500 with paired-end: 2. 75 bp Transcriptome was assembled from RNA-Seq fastq files using Trinity v2.1.1 ( Ref.3) with default parameters. A BLAST database was created with the assembled transcripts using makeblastdb application from ncbi-blast+ (v2.2.31+). The sequences of transcripts of interest were then blasted against this database using blastn application from ncbi-blast+ ( Ref.4) with parameter words size 7 .

RESUlts

\begin{tabular}{|l|l|c|c|c|c|c|c|c|c|}
\hline ueryID & $\begin{array}{l}\text { Query } \\
\text { Name }\end{array}$ & SubjectID & $\begin{array}{c}\text { Identity } \\
(\boldsymbol{\%})\end{array}$ & Length & Mismatch & Gapopen & $\begin{array}{c}\text { Query } \\
\text { cover } \\
(\boldsymbol{\%})\end{array}$ & E-value & Bitscore \\
\hline NM_000545.8 & HNF1A & TRINITY_DN8044_c1_g1_i1 & 78,81 & 118 & 25 & 0 & 3,00 & $5,00 \mathrm{E}-14$ & 80,50 \\
\hline
\end{tabular}

The table 1 summarizes the characteristics of the O. nigra HNF1A transcriptome The sequence in 5 '-3' is the following one :

\section{TRINITY_DN8044_c1_g1_i1 (HNF1A)}

5'TGAAGATGATGAACAGTTACCTGATTAT
GGGCCAATGGATACAGGATCATTAAGGG
ACCA
ATTGCTAAGGCAAGATTCTTGGCAAGTTG
CCAAGATGATCAAGACGTATATGCAACAG
CA
CAACATCCCACAACGTGAAGTTGTAGAC
GCTACAGGCCTGAATCAGAGTCACCTATC
ACA

ACATCTCAACAAGGGCACGCCAATGAAG AG 3'

\section{Discussion- CONCLUSION}

The sequence of the HFN1A transcriptome is short in 5'-3' but it seems true that the gene is present in Invertebrates : how surprising it is !

Since we have discovered the IPA (Invertebrate Primitive Antibody), many genes we find in Human are revealed in Echinodermata Phylum. 
We cite : PECAM1 gene ( Ref 5), CXCR4 gene (Ref.6), ICAM1 gene (Ref.7)

All these genes are classified in Cells Showing the antigen. They are found in Human.

According G. Salzano et al (Ref8) : «

The HNF1A gene is located at chromosome $12 \mathrm{q} 24$, comprises 10 exons that span $23,790 \mathrm{bp}$, and encodes a 631-amino-acid protein which is expressed in many tissues such as the liver, kidney, and pancreas (Ref .9). In pancreatic beta cells, HNF1A plays a regulatory role in the expression of several genes involved in glucose metabolism and transport, such as glucose transporter 2 (GLUT2) and pyruvate kinase. One of the first direct studies of human islets from an individual with a heterozygous missense variant in the HNF1A locus confirmed that loss-of-function variants of HNF1A lead to insulin-insufficient diabetes not through a significant loss of beta-cell mass but rather by impacting beta-cell transcriptional regulatory networks, resulting in the impairment of betacell pathways that are needed for a normal insulin response to glucose. Heterozygous mutations of HNF1A that cause diabetes were first identified in 1996, and more than 400 different mutations have since been described. These mutations include missense and nonsense mutations, insertions and duplications, deletions, insertion/deletions, promoter region mutations, and splice site mutations. These mutations occur throughout the gene, but they are mainly found in exon 2 ( 0.33 mutations per nucleotide) and in exon 4 ( 0.28 mutations per nucleotide). A high proportion of the mutations, particularly missense mutations, involve the dimerization and DNA-binding domains, which seem to be less tolerant of minor structural changes. The type and the location of the mutation seem to be linked to the age at diabetes diagnosis. Mutations involving the first six exons are correlated with an earlier diagnosis (median age of 18 years) than mutations located in either exons $8-10$ or the transactivation domain (median age of 30 years). Patients with truncating mutations are diagnosed at an average age of 20 years regardless of the location of the mutation with in the HNF1A gene »

The gold standard method of screening for mutations is sequence analysis of the coding regions and conserved splice sites. In patients with a very specific clinical phenotype, Sanger sequencing, also called the chain termination method, may be sufficient to identify abnormalities of the selected gene. The recent introduction of targeted next-generation sequencing (NGS) - which permits the sequencing of gene collections, exomes, or even whole genomes - has led to an increase in the number of patients diagnosed with monogenic diabetes.

Echinodermata don't possess pancreas and liver but elaborated digestive coeca. They have also a sophisticated immune system which presents all the characteristics of adaptative immunity. In that, they may be compared to humans.

\section{REFERENCES}

[1] Leclerc. M, et al(2018) Int. J. Vaccines Vaccin 5 (1) : 00095

[2] Leclerc. M, et al(2018) Cell . Cellular Life. Sci. J 3(1) 000117

[3] Grabher.M.G et al (2011) Nature Biotechnology 29 644-652

[4] Altschul. S.F et al (1990) J.Mol.Biol 215(3) 403-410

[5] Leclerc, M. (2020) Immunology,submitted.

[6] Leclerc.M (2020) Immunology, submitted

[7] Leclerc.M (2020) Immunology, submitted

[8] Salzano. G et al (2019) Diabetes Therapy 10 1543-1548

[9] Pontoglio. M et al (1996) Cell 84 575-585

Citation: Michel Leclerc, "Evidence of Hnfla Gene in Invertebrates: The Echinodermata", International Journal of Research Studies in Medical and Health Sciences. 2020; 5(1): 01-02.

Copyright: () 2020 Michel Leclerc, This is an open-access article distributed under the terms of the Creative Commons Attribution License, which permits unrestricted use, distribution, and reproduction in any medium, provided the original author and source are credited. 